\title{
Valor pronóstico de la respuesta patológica a la radioquimioterapia preoperatoria en el cáncer de recto bajo localmente avanzado*
}

\author{
Drs. GUILLERMO BANNURA C. ${ }^{1}$, CLAUDIO VARGAS N. ${ }^{1}$, \\ ALEJANDRO BARRERA E. ${ }^{1}$, CARLOS MELO L. ${ }^{1}$, FELIPE ILLANES F. ${ }^{1}$
}

\author{
Servicio y Departamento de Cirugía, Hospital Clínico San Borja Arriarán. Campus Centro, Facultad de Medicina, \\ Universidad de Chile. \\ Santiago, Chile.
}

\begin{abstract}
\section{Prognostic value of pathological response to chemo radiotherapy of locally advanced low rectal cancer}

Background: Preoperative chemo radiotherapy improves the prognosis of locally advanced low rectal cancer and induces a pathological response in the tumor, which may have prognostic value. Aim: To assess the results of rectal cancer treatment according to the degree of pathological response of the tumor after chemo radiotherapy. Patients and Methods: All patients with a locally advanced rectal cancer located within $11 \mathrm{~cm}$ of the rectal margin, subjected to preoperative chemo radiotherapy followed by surgical treatment in a period of 13 years, were included. Pathological response was classified as complete, intermediate and poor. The tumor was staged according to TNM 2002 classification. Survival was analyzed with Kaplan Meier curves and Cox regression. Results: Patients were followed for a mean of 50 months (range 18-156). Exclusive and global local relapse was observed in 3 and $9.6 \%$ of patients, respectively. Pathological response was complete in 13 patients (none died), intermediate in 23 (three died) and poor in 68 (22 died). Global five years survival was $74 \%$. There was a concordance of 0.64 between survival and pathological response. The concordance between survival and TNM classification was 0.69 . Conclusions: The pathological response of the tumor to chemo radiotherapy has a good concordance with prognosis, although it is not superior to the final pathological status.

Key words: Rectal cancer, prognosis, chemo radiotherapy.
\end{abstract}

\section{Resumen}

Introducción: La radioquimioterapia (RQT) preoperatoria en el manejo del cáncer de recto bajo localmente avanzado mejora el control locoregional y es capaz de inducir en el tumor una respuesta patológica (RP) variable que podría tener implicancia pronóstica. El objetivo de este estudio es evaluar el grado de RP inducida por la RQT y comparar los resultados oncológicos de acuerdo al grado de RP luego de RQT neoadyuvante. Pacientes y Método: Se incluyen todos los pacientes con un tumor de recto localmente avanzado por

* Recibido el 22 de octubre de 2012 y aceptado para publicación el 15 de noviembre de 2012.

Los autores no refieren conflictos de interés.

Correspondencia: Dr. Guillermo Bannura C.

Las Limas 1622, Santiago, Chile.

gbannura@vtr.net 
debajo de los $11 \mathrm{~cm}$ al margen anal sometidos a RQT seguida de cirugía radical con intención curativa en un período de 13 años. La RP fue categorizada como completa, intermedia y pobre. Para la etapificación patológica se utilizó la clasificación TNM 2002. Las curvas de sobrevida fueron estimadas según Kaplan-Meier, se empleó el modelo de regresión de Cox para el análisis multivariado y los coeficientes de concordancia fueron evaluados según el estadístico $C$ de Harrell y el $K$ de Gonen-Heller. Resultados: Seguimiento promedio 50 meses (extremos 18-156). La recidiva local exclusiva fue $3 \%$ y la recidiva local global fue 9,6\%. La RP fue completa en 13 pacientes (no fallecidos), Intermedia (ypT1-T2N0) en 23 (3 fallecidos) y fue pobre (ypT3/T4 y/o LN+) en 68 (22 fallecidos). Sobrevida global a 5 años 74\%. Hubo una fuerte correlación entre la sobrevida y la RP, con un coeficiente de concordancia $(0,64)$ ligeramente inferior al coeficiente de la etapificación patológica definitiva de acuerdo al TNM $(0,69)$. Conclusión: El grado de RP es un marcador que se correlaciona bien con el pronóstico oncológico con un índice de concordancia de 0,69 cuando se asocia con la localización del tumor, aunque no supera al estadio patológico final que alcanza un valor de 0,74.

Palabras clave: Cáncer recto, radioquimioterapia, sobrevida, respuesta patológica.

\section{Introducción}

La radioquimioterapia (RQT) preoperatoria seguida de cirugía radical es en la actualidad el tratamiento estándar del adenocarcinoma del recto bajo localmente avanzado. Se considera como tal a los tumores cuyo límite inferior se localiza por debajo de los $11 \mathrm{~cm}$ desde el margen anal medido con un instrumento rígido y cuyo compromiso de la pared intestinal sobrepasa la muscular propia y/o con linfonodos metastásicos ( $\mathrm{LN}+)$ (de acuerdo a la clasificación TNM corresponden a tumores T3/T4 y/o $\mathrm{N}+$ en la etapificación clínica) $)^{1-3}$. La RQT produce en mayor o menor medida una disminución del número de $\mathrm{LN}+($ factor $\mathrm{N}$ ) y grados variables de reducción del compromiso transmural (factor T), fenómeno que se conoce como sub-etapificación (tumor downstaging). Los cambios producidos por la RQT en el tumor varían desde una respuesta patológica nula hasta una respuesta patológica completa (RPC), es decir, la ausencia de células tumorales viables. Aunque controversial, el significado pronóstico de esta respuesta patológica para algunos autores es un factor pronóstico relevante y tiene la ventaja de ser una información que se obtiene precozmente luego de la cirugía radical ${ }^{4}$. El objetivo de este estudio es comparar los resultados oncológicos a largo plazo de los pacientes con cáncer de recto localmente avanzado sometido a neoadyuvancia con QRT más cirugía radical, estratificados de acuerdo al grado de respuesta patológica a la RQT en comparación con la etapificación patológica.

\section{Pacientes y Método}

En el mes de junio de 1999 iniciamos un protocolo prospectivo no aleatorio de neoadyuvancia en cáncer de recto localmente avanzado seguido de cirugía radical. Se incluye en el protocolo a todos los pacientes portadores de un adenocarcinoma ubicado en el recto inferior $(0-7 \mathrm{~cm}$ del margen anal) $\mathrm{y}$ en el recto medio $(7-11 \mathrm{~cm})$ cuyo borde inferior esté por debajo de $11 \mathrm{~cm}$ medido mediante con un rectoscopio rígido. La etapificación clínica se efectuó mediante el examen clínico (tacto rectal), tomografía computada (TC) de tórax, abdomen y pelvis, complementados con resonancia magnética (RM) de pelvis y/o ultrasonografía endorrectal (USER) en casos de mayor complejidad o frente a evaluaciones clínicas contradictorias. Se incluyen los tumores T3/T4 y/o N+ que completaron el esquema de neoadyuvancia con RQT de acuerdo al esquema largo (45 Gray en 5 semanas 25 sesiones) más una sobreimpresión pélvica para alcanzar 50,4 Gray, asociado a quimioterapia con 5-FU en dosis de $600 \mathrm{mg} / \mathrm{m}^{2}$ en infusión continua durante la primera y la quinta semana del tratamiento radiante. Luego de obtenida la cicatrización de las heridas y la recuperación del estado general, se indicó 6 ciclos de 5 -FU en dosis de $450 \mathrm{mg} / \mathrm{m}^{2}$ más leucovorina 20 $\mathrm{mg} / \mathrm{m}^{2}$ por 5 días cada 28 días en los pacientes cuya etapificación patológica definitiva fuera estadio III $(\mathrm{LN}+)$. Se excluyen los pacientes con tumores T1/ $\mathrm{T} 2$, los casos sometidos a resección local luego de neoadyuvancia, los tumores en estadio IV, los tumores ubicados en recto superior $(11-15 \mathrm{~cm}$ del margen anal) y los pacientes con un cáncer de recto bajo en el contexto de una poliposis o con un cáncer colorrectal sincrónico.

La etapificación se realizó de acuerdo a la sexta edición de la clasificación AJCC conocida como TNM 2002 de la UICC 5 . Se define como RPC la completa regresión del tumor (ausencia de células neoplásicas viables, es decir, en la nomenclatura TNM equivale a ypT0N0) e incluye a los pacientes con un espécimen que muestra lagos de mucina acelulares. La respuesta patológica intermedia (RPI) se define como una mejoría en la estadificación clínica hasta un estadio ypT1-T2N0 (se reconoce tumor viable que compromete la submucosa (T1) o hasta la capa muscular (T2) sin LN+). Las lesiones residuales ypT3/T4 y/o cualquier $\mathrm{T}$ con $\mathrm{LN}+$ se 
define como repuesta patológica pobre (RPP). La cirugía radical se programó entre 8 y 12 semanas luego de terminada la neoadyuvancia y consiste en la resección del rectosigmoides con extirpación total del mesorrecto por medio de una de cirugía abierta o laparoscópica, con ligadura alta de la arteria mesentérica inferior, preservando el plexo hipogástrico. En casos límites, la toma de decisiones sobre la conservación esfinteriana fue intraoperatoria. Todas las anastomosis fueron efectuadas con suturas mecánicas con la técnica de grapado doble y los pacientes con una anastomosis por debajo de 5-6 $\mathrm{cm}$ fueron protegidos con una ileostomía en asa. Se define como recidiva local la reaparición de tumor en la pelvis y como recidiva a distancia la carcinomatosis peritoneal y/o la presencia de metástasis en hígado o pulmón.

Para el análisis estadístico se utilizó la prueba de $\chi^{2}$ de Pearson y el $t$ test de Student para determinar asociaciones entre dos variables, según corresponda. Las curvas de sobrevida fueron estimadas según el método de Kaplan-Meier y para comparar las curvas de sobrevida se empleó el test log-rank. El modelo de regresión de Cox se utilizó para el análisis multivariado. La probabilidad de que las predicciones y los resultados sean concordantes se evaluó mediante los coeficientes de concordancia del estadístico $C$ de Harrell y el $K$ de Gonen-Heller (un coeficiente cercano a 1 refleja un modelo con una discriminación casi perfecta). Se consideró estadísticamente significativo un $\mathrm{p} \leq 0,05$.

\section{Resultados}

En un período de 13 años se intervinieron en forma consecutiva 104 pacientes portadores de un tumor de recto bajo localmente avanzado sometidos a neoadyuvancia más cirugía radical con intención curativa, con una edad promedio de 60,7 años (extremos 27-84), el 54\% mujeres, el 47\% con enfermedades asociadas relevantes, destacando la hipertensión arterial en el $28 \%$ y la diabetes mellitus en el 6\%. Para la etapificación clínica se empleó el TC de tórax, abdomen y pelvis en el $100 \%$ de los casos, la USER en el $20 \%$ y la resonancia magnética de pelvis en el $4 \%$. El antígeno carcinoembrionario (CEA) promedio de la serie previo a la neoadyuvancia fue de $13,1 \mathrm{ng} / \mathrm{ml}$ (extremos $0,7-566$ ) y el $36 \%$ de los casos tenía un CEA $>5 \mathrm{ng} / \mathrm{ml}$. El intervalo entre la RQT y la cirugía radical fue en promedio de 14,5 semanas (extremos 6-25) y en el $45 \%$ de los casos fue superior a 12 semanas. La resección radical se realizó con conservación esfinteriana en $74(71 \%)$ de los casos y la anastomosis quedó en promedio a $4,8 \mathrm{~cm}$ del margen anal (extremos 1-8).
El tiempo de hospitalización promedio fue de 14 días (extremos 4-98). La morbilidad global afectó al $37 \%$ de los pacientes, se reoperan 4 y la dehiscencia anastomótica clínica fue 4,8\% (5 de 74 pacientes). No hubo mortalidad operatoria en esta serie.

La etapificación patológica reveló una RPC en 13 pacientes y casi completa en otros 7 . El número promedio de ganglios examinados fue de 8,3 (extremos $0-29)$ y el número de $\mathrm{LN}+$ promedio fue 0,75 (extremos 1-20) presentes en 25 pacientes estadio III. La distribución de los pacientes de acuerdo a la etapificación definitiva se aprecia en la Tabla 1. La categorización según respuesta clínica a la RQT preoperatoria y la censura se señala en la Tabla 2. La asociación entre el compromiso transmural (factor $\mathrm{T}$ ), el compromiso linfonodal $(\mathrm{N})$ y la censura se detalla en la Tabla 3.

Tabla 1. Etapificación definitiva. TNM $20026^{\circ}$ edición

\begin{tabular}{|lrcc|}
\hline Estadio & n & $\mathbf{( \% )}$ & Fallecidos (\%) \\
0 (RPC) & $13(12,5)$ & 0 \\
I (T1-T2 N0) & $23(22,1)$ & $2(8,7)$ \\
IIA (T3 N0) & $38(36,5)$ & $10(26,3)$ \\
IIB (T4 N0) & 5 & $(4,8)$ & $1(20)$ \\
IIIA (T1-T2 N1) & 5 & $(4,8)$ & $2(40)$ \\
IIIB (T3-T4 N1) & $14(13,5)$ & $6(43)$ \\
IIIC (T1-T4 N2) & 6 & $(5,8)$ & $4(67)$ \\
Total & $104(100)$ & $25(24)$ \\
\hline
\end{tabular}

Tabla 2. Categorización según RP y censura

\begin{tabular}{|lrcc|}
\hline RP & n & \% & $\begin{array}{c}\text { Censura } \\
\text { n (\%) }\end{array}$ \\
Completa & 13 & 12,5 & 0 \\
Intermedia (T1-T2 N0) & 23 & 22,1 & $3(13)$ \\
Pobre (T3-T4 y/o N+) & 68 & 65,4 & $22(32,3)$ \\
Total & 104 & 100 & $25(24)$ \\
\hline
\end{tabular}

Tabla 3. Asociación entre el factor $\mathrm{T}$, factor $\mathbf{N}$ y censura

\begin{tabular}{|crccc|}
\hline T & n & LN (+)/LN (-) & \% LN + & Censura \\
yрT0 (RPC) & 13 & 0 & 0 & 0 \\
ypT1 & 9 & $1 / 8$ & 11 & 0 \\
ypT2 & 19 & $4 / 15$ & 21 & 3 \\
yрT3 & 55 & $17 / 38$ & 69 & 20 \\
ypT4 & 8 & $3 / 5$ & 37,5 & 2 \\
\hline
\end{tabular}




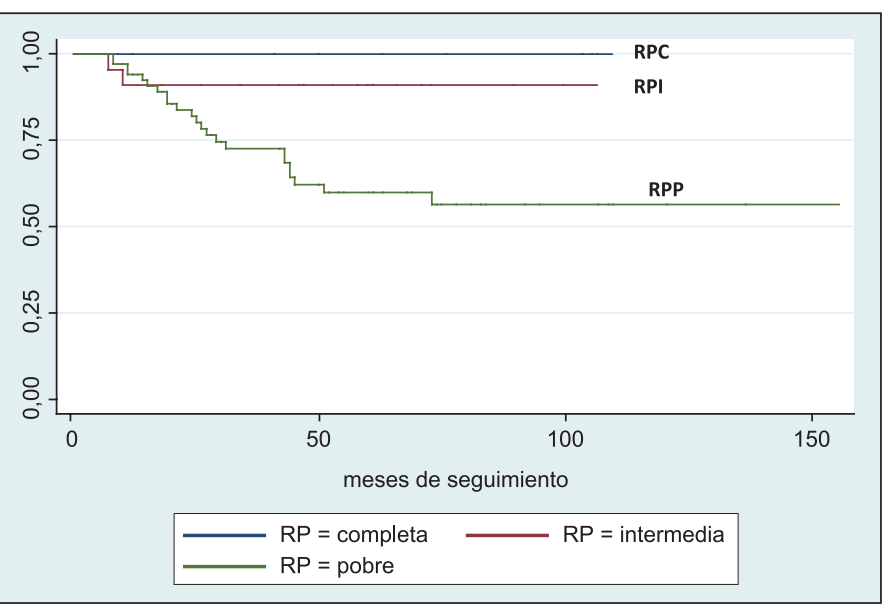

Figura 1. Curvas de sobrevida de Kaplan-Meier según respuesta patológica.

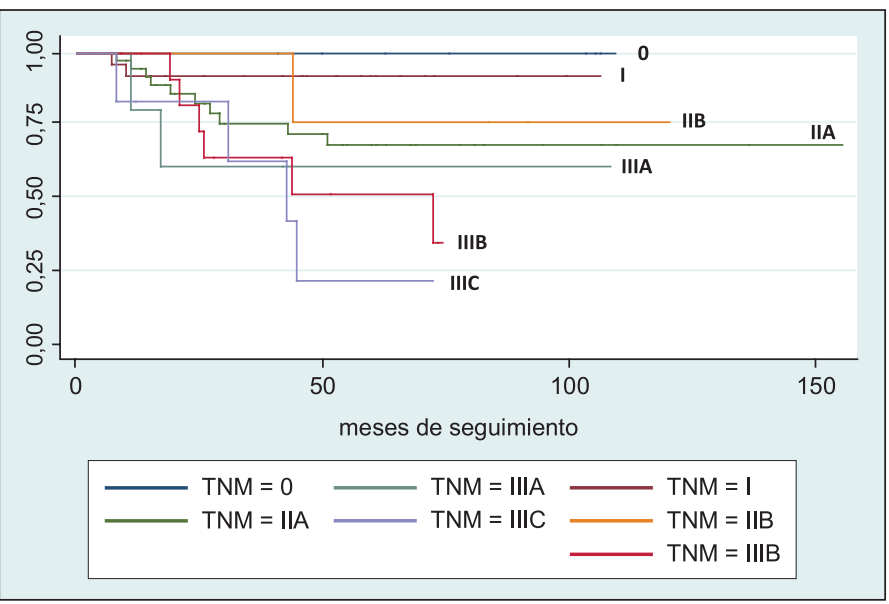

Figura 2. Curvas de sobrevida de Kaplan-Meier según etapificación. TNM 2002.

Tabla 4. Modelo de Regresión de Cox: análisis univariado

\begin{tabular}{|lccc|}
\hline Variable & Hazard ratio & $\mathbf{p}$ & IC 95\% \\
Edad $(<65$ años $)$ & 1,01 & 0,53 & $0,97-1,04$ \\
Género $(\mathrm{m} / \mathrm{h})$ & 0,70 & 0,38 & $0,31-1,56$ \\
Hematocrito $(<37,5 \%)$ & 0,94 & 0,15 & $0,87-1,02$ \\
Albúmina $(<4 \mathrm{gr} / \mathrm{dl})$ & 1,14 & 0,74 & $0,51-2,57$ \\
CEA $(<5 \mathrm{ng} / \mathrm{ml})$ & 0,99 & 0,73 & $0,99-1,00$ \\
Localización (recto inferior) & 2,53 & 0,02 & $1,11-5,75$ \\
Diferenciación (grados 1 y 2) & 0,67 & 0,70 & $0,09-4,99$ \\
Mucina & 2,12 & 0,07 & $0,93-4,81$ \\
PVL y/o PN & 2,77 & 0,018 & $1,18-6,48$ \\
Tamaño (>3 cm) & 1,18 & 0,068 & $0,98-1,41$ \\
Factor T & 2,84 & 0,01 & $1,26-6,40$ \\
\hline
\end{tabular}

Tabla 5. Test de concordancia

\begin{tabular}{|lcc|}
\hline Variable & $\begin{array}{c}\text { Harrell's } \\
\text { C }\end{array}$ & $\begin{array}{c}\text { Gonen- } \\
\text { Heller }\end{array}$ \\
TNM & 0,69 & 0,73 \\
$\begin{array}{l}\text { RP (respuesta } \\
\text { patológica) }\end{array}$ & 0,64 & 0,69 \\
$\begin{array}{l}\text { Localización (Recto } \\
\text { medio/inferior) }\end{array}$ & 0,61 & 0,65 \\
$\begin{array}{l}\text { Localización + TNM } \\
\text { Localización + RP }\end{array}$ & 0,74 & 0,76 \\
\hline
\end{tabular}

El seguimiento global promedio de la serie fue de 50 meses (extremos 18-156). El seguimiento global promedio de los 25 pacientes fallecidos por la enfermedad fue de 26,7 meses (extremos 7-53). Hubo 3 pacientes con una recidiva local exclusiva, de los cuales se reoperan 2 con sobrevida de 107 y 28 meses, respectivamente. Hubo 7 pacientes con recidiva local y a distancia y 17 pacientes con recidiva a distancia exclusiva. La recidiva local global fue 9,6\%. La curva de sobrevida según Kaplan-Meier de acuerdo a la etapificación TNM 2002 y según la respuesta patológica, se aprecian en las Figuras 1 y 2. En el análisis univariado, la localización del tumor (recto inferior/medio), la permeación vascular linfática y/o perineural y el compromiso transmural alcanzan significación estadística (Tabla 4). Al introducir las variables en el análisis multivariado (modelo de regresión de Cox), sólo la localización del tumor, el TNM y la RP alcanzan significación estadística. El coeficiente de concordancia de ambos test fue ligeramente superior al cruzar la variable localización con el TNM comparado con la localización y la RP (Tabla 5).

\section{Discusión}

En esta serie se confirma el valor de la neoadyuvancia más cirugía radical en el manejo multimodal del cáncer de recto localmente avanzado, logrando una RPC o casi completa en el $20 \%$ de los casos y una reducción global del es- 
tadio clínico en el 35\%. Este downstaging patológico se refleja en la distribución de los pacientes en los estadios TNM (Tabla 1), con una discreta desviación hacia estadios menos avanzados al comparar los resultados de pacientes resecados sin neoadyuvancia ${ }^{6}$. Esta subetapificación podría relacionarse con el pronóstico, ofreciendo una información predictiva inmediata luego de la cirugía radical y, eventualmente, orientar la terapéutica adyuvante. En nuestra serie no hemos tenido recidiva local en pacientes con una RPC o casi completa, lo que en series más numerosas no supera el 2,6\% ${ }^{4,7}$. En el seguimiento a largo plazo en este escenario los pacientes muestran una sobrevida libre de enfermedad entre 88 y $100 \%{ }^{2,4,7,8}$. Desgraciadamente, la RP es pobre en dos tercios de los pacientes, con una tasa relativamente alta de recidiva local $(10 \%)$ y de mortalidad por cáncer en este subgrupo de pacientes $(25 \%)$, similar a otras series de mayor envergadura ${ }^{4}$. Sin duda, el mayor desafío de esta estrategia multimodal en el futuro mediato es la identificación de las variables o factores predictivos de la respuesta favorable en los pacientes respondedores, aspecto altamente controversial en la literatura especializada. En nuestra experiencia y la de otros ${ }^{8-10}$, la negativización de los linfonodos muestra una marcada inflexión positiva en la curva de supervivencia, como se aprecia en las Figuras 1 y 2.

Aunque el TC no es el método ideal para la etapificación clínica del cáncer de recto bajo, este examen más el examen clínico permite confirmar que efectivamente se trata de una serie de pacientes con un cáncer de recto localmente avanzado. La asociación entre el compromiso transmural del tumor (factor $\mathrm{T}$ ) y el compromiso linfonodal $(\mathrm{N})$ en los pacientes sometidos a RQT (Tabla 3) ha sido controversial en la literatura actual. Algunos autores han postulado que el compromiso linfonodal según la profundidad del tumor se altera luego de la RQT ${ }^{11}$, sugiriendo que el impacto de la RQT sería mayor en los ganglios que en el tumor primario. Al comparar pacientes sin RQT intervenidos con cirugía radical, comprobamos que hay una significativa reducción del tamaño promedio del tumor, del número promedio de linfonodos recolectados y del número promedio de $\mathrm{LN}+{ }^{6}$. En acuerdo con otros autores ${ }^{4,12}$, nuestros resultados confirman que el compromiso transmural residual luego de neoadyuvancia se correlaciona fuertemente con el compromiso linfonodal, el que progresa a medida que aumenta el factor $\mathrm{T}$. Ello reduce en forma significativa las indicaciones de resección local luego de RQT, situación que se plantea en pacientes de alto riesgo quirúrgico o en aquellos con riesgo de perder el aparato esfinteriano.

Es claro que la variable RP se relaciona fuertemente con la etapificación patológica definitiva según el TNM, lo que queda en evidencia al observar las curvas de sobrevida según ambas categorías (Figuras 1 y 2), pero indudablemente no son lo mismo. Como se muestra en este estudio, la RPC corresponde exactamente al estadio TNM 0 y la respuesta intermedia (T1-T2, N0) corresponde precisamente al estadio TNM I, lo que significa que se incluyen en ambas categorías pacientes comparables o equivalentes. Por el contrario, la categoría RP pobre incluye pacientes con una etapificación TNM muy variable, que varía entre pacientes en estadio IIA hasta el estadio IIIC, es decir, la categoría RPP mezcla estadios II y III de muy diferente pronóstico y tampoco reconoce los casos poco frecuentes de pacientes T1-T2 con LN+ (antiguamente AsterColler C1). Ello contradice el valor pronóstico del compromiso ganglionar luego de neoadyuvancia en el cáncer de recto bajo localmente avanzado y se refleja parcialmente en los resultados de los Test de concordancia de Harrell y de Gonnen-Heller aplicados en este estudio, con una ventaja significativa para el TNM en cuanto al grado de robustez pronóstica.

En conclusión, en esta serie de 104 pacientes portadores de un cáncer de recto bajo localmente avanzado diagnosticados mediante el examen clínico con ayuda de TC sometidos a terapia multimodal, se confirma el valor pronóstico de las variables localización del tumor, el compromiso en la pared intestinal (T) y los linfonodos $(\mathrm{N})$, factores ampliamente reconocidos en la literatura especializada en pacientes sin RQT preoperatoria. El coeficiente de concordancia de la RP es similar al encontrado en otros estudios que han empleado una categorización diferente de la RP9 . El valor pronóstico de la RP no logra superar la fortaleza de la etapificación definitiva luego de RQT en esta serie, hecho que tiene defensores $^{9}$ y retractores ${ }^{4}$, lo que amerita mayor estudio $^{13,14}$.

\section{Referencias}

1. Sauer R, Becker H, Hohenberger W, Rödel C, Wittekind $\mathrm{C}$, Fietkau R, et al. Preoperative versus postoperative chemoradiotherapy for rectal cancer. $\mathrm{N}$ Engl J Med. 2004;351:1731-40.

2. Fleming FJ, Pählman L, Monson JRT. Neoajuvant therapy in rectal cancer. Dis Colon Rectum 2001;54:901-12.

3. Garajová I, Di Girolamo S, de Rosa F, Corbelli J, Agostini V, Biasco G, Brandi G. Neoadjuvant treatment in rectal cancer. Chemother Res Pract. 2011;2011:839742. Epub 2011 Sep 21.

4. Park IJ, You N, Agarwal A, Skibber JM, RodríguezBigas M, Eng K, Feig BW, et al. Neoadjuvant treatment response as an early response indicator for patients with 
rectal cancer. J Clin Oncol. 2012;30:1770-6.

5. Greene FL, Page DL, Fleming ID. American Joint Committee on Cancer. Cancer staging manual. ${ }^{\text {th }}$ edition. New York, NY: Springer-Verlag, 2002.

6. Bannura G, Barrera A, Melo C, Contreras J, Soto D. Resultados del tratamiento con neoadyuvancia del cáncer de recto localmente avanzado. Rev Chil Cir. 2010;62:607-13.

7. Martin ST, Heneghan HM, Winter DC. Systematic review and meta-analysis of outcomes following pathological complete response to neoadjuvant chemoradiotherapy for rectal cancer. Br J Surg. 2012;99:918-28.

8. Yeo SG, Kim DY, Kim TH, Chang HJ, Oh JH, Park W, et al. Pathologic complete response of primary tumor following preoperative chemoradiotherapy for locally advanced rectal cancer: long term outcomes and prognostic significance of pathologic nodal status. Ann Surg. 2010;252:998-1004.

9. Quah H-M, Chou JF, Gonen M, Shia J, Schrag D, Saltz LB, et al. Pathologic stage is most prognostic of disease-free survival in locally advanced rectal cancer patients after preoperative chemoradiation. Cancer 2008;113:57-64.
10. Kim NK, Kim Y-W, Min B-S, Lee K-Y, Sohn S-K, Cho $\mathrm{C}-\mathrm{H}$. Factors associated with local recurrence after neoadjuvant chemoradiation with total mesorectal excision for rectal cancer.World J Surg. 2009;33:1741-9.

11. Medich D, McGinty J, Parda D, Karlovits S, Davis C, Caushaj P, et al. Preoperative chemoradiotherapy and radical surgery for locally advanced distal adenocarcinoma: pathologic findings and clinical implications. Dis Colon Rectum 2001;44:1123-28.

12. Kim D-W, Kim DY, Kim TH, Jung KH, Chang HJ, Sohn $\mathrm{S}-\mathrm{K}$, et al. Is T classification still correlated with lymph nodes status after preoperative chemoradiotherapy for rectal cancer? Cancer 2006;106:1694-700.

13. Bonnetain F, Bosset JF, Gerard JP, Calais G, Conroy T, Mineur L, et al. What is the clinical benefit of preoperative chemoradiotherapy with 5FU/leucovorin for T3-4 rectal cancer in a pooled analysis of EORTC 22921 y FFCD 9203 trials: Surrogacy in question? Eur J Cancer 2012;48:1781-90.

14. Rodel C, Martus P, Papadoupolos T, Füzesi L, Klimpfinger M, Fietkau R, et al. Prognostic significance of tumor regression after preoperative chemoradiotherapy for rectal cancer. J Clin Oncol. 2005;23:8688-96. 